\title{
Angular distribution of photoelectrons ejected from HI by polarised coherent vUV radiation
}

\author{
A Mank, M Drescher, T Huth-Fehre, G Schönhense, N Böwering and \\ U Heinzmann \\ Universität Bielefeld, Fakultät für Physik, D-4800 Bielefeld, Federal Republic of Germany
}

Received 30 May 1989

\begin{abstract}
The asymmetry parameter $\beta$ of the photoelectron angular distribution of the process $\mathrm{HI}\left(5 \pi^{4}\right)^{1} \Sigma^{+}\left(v^{\prime \prime}=0\right)+h \nu \rightarrow \mathrm{HI}^{+}\left(5 \pi^{3}\right){ }^{2} \Pi_{3 / 2}\left(v^{\prime}=0\right)+\mathrm{e}^{-}$was measured at photon energies from 86440 to $87000 \mathrm{~cm}^{-1}$. The data show resonance structure due to autoionisation that was not resolved before. The results are compared with predictions of an $a b$ initio calculation by $\mathrm{H}$ Lefebvre-Brion and co-workers.
\end{abstract}

Until recently, experiments studying the dynamics of molecular photoionisation were restricted by the limited resolution of conventional vacuum-ultraviolet (VUV) radiation sources. Experiments using multiphoton ionisation techniques can have the high resolution necessary for a detailed examination of molecular electronic structure, but they are governed by a different set of selection rules compared with single-photon photoionisation. Therefore, the dynamics of the process is different for the two cases. With the development of non-linear frequency-mixing techniques (Hilbig and Wallenstein 1983) high-power, tunable, narrow-band, coherent vuv radiation is now available for one-photon photoionisation experiments. Results of photoemission-yield measurements have demonstrated the advantages of this light source (Huth et al 1988, Hart and Hepburn 1989).

It is the purpose of this letter to report the first results on the angular distribution symmetry parameter of the photoelectron intensity obtained by use of narrow-band, coherent vUV radiation. In this study, $\mathrm{HI}$ was selected as a target and single-photon transitions into the autoionisation region between the spin-orbit split ${ }^{2} \Pi$ ionic ground states were investigated. For HI, ab initio calculations of the dynamical photoionisation parameters in the autoionisation region are available (Lefebvre-Brion et al 1985). The total photoelectron yield in this region was investigated recently (Huth et al 1988) and the angular distribution of the photoelectrons was measured using synchrotron radiation with a bandwidth of 0.7 to $1.3 \AA$ by Carlson et al (1986). These $\beta$ measurements could not reproduce, however, the detailed structure predicted by the ab initio calculation; thus the question remained whether this was due to a lack of experimental resolution or due to physical structure not taken into account in the theory.

The vUV radiation was generated by non-linear sum-frequency mixing of pulsed dye-laser radiation in mercury vapour (Huth et al 1988). The mixing process involved radiation from two dye lasers, one of which was frequency doubled in a KDP crystal and tuned to the $6 \mathrm{~s} \rightarrow 6 \mathrm{~d}$ two-photon resonance in mercury at $71333.180 \mathrm{~cm}^{-1}$, giving a strong resonant enhancement of the mixing process. The other dye laser could be 
scanned from the visible to the near-infrared region of the spectrum. The resulting tuning range in the vuv was from 83500 to $88500 \mathrm{~cm}^{-1}$. The intensity of the vuv radiation produced was measured to be higher than $10^{10}$ photons/pulse at a repetition rate of $11 \mathrm{~Hz}$. The bandwidth of the vUv radiation was determined to be $0.38 \mathrm{~cm}^{-1}$. Using a four-mirror analyser, the linear polarisation of the vUv source was measured to be better than $90 \%$, depending on the quality of the lithium fluoride (LiF) optics used. The electric field vector of the linear polarisation of the vuv could be rotated by rotating the $\boldsymbol{E}$ vector of both incoming dye-laser beams with a $\lambda / 2$ Fresnel rhomb. The energy scale of the vUv radiation was checked by detection of $I_{2}$ fluorescence, optogalvanic spectra of a neon-filled hollow-cathode lamp and an interferometer scan control to fill in the gaps between the resonances used for calibration. Combining these methods, the energy of the Vuv radiation was known absolutely within an accuracy of $0.2 \mathrm{~cm}^{-1}$. Behind the conversion cell the vUv radiation generated was separated from the fundamental laser wavelength and other mixing products by means of a $\mathrm{LiF}$ prism and then guided to the target. A detailed description of the radiation source is given elsewhere (Huth et al 1988).

The set-up of the angle-resolved photoelectron experiment is shown in figure 1. The vuv radiation crosses a molecular beam from a focusing capillary array (Lucas 1973) used as a gas inlet. The intensity of the vuv radiation is monitored by a vacuum photodiode for normalisation of the photoelectron intensity data. The photodiode can be moved out of the beam axis in order to analyse the polarisation of the vuv by use of a four-mirror reflecting analyser.

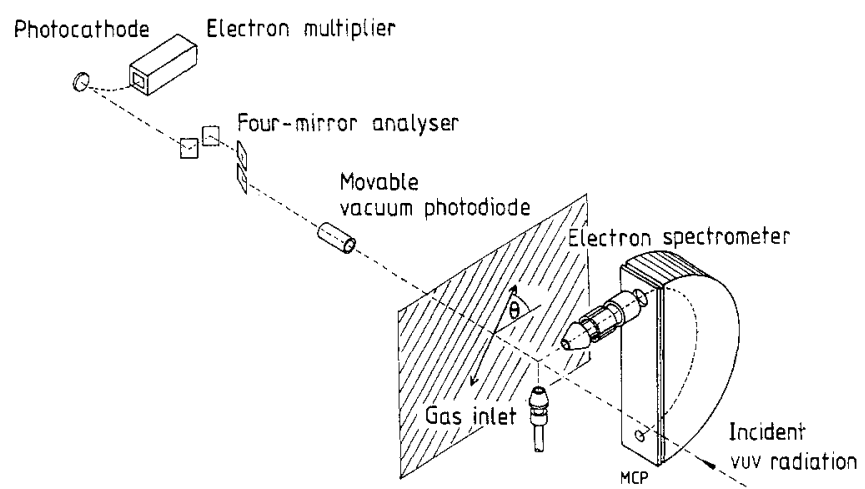

Figure 1. Experimental set-up for angle-resolved photoelectron spectroscopy. The spectrometer analyses electrons ejected perpendicularly to the axis of the incident radiation. The $\boldsymbol{E}$-vector of the linear polarisation of the VUV radiation can be rotated around the beam axis. The intensity of the VUV is monitored by a vacuum photodiode; alternatively, the polarisation can be determined by a four-mirror analyser.

The photoelectrons emitted perpendicular to the axis of the vUV are energy analysed in a simulated hemispherical spectrometer (Jost 1979) with a mean path radius of $75 \mathrm{~mm}$. The angular resolution of this set-up is $\theta= \pm 7.5^{\circ}$. The spectrometer is used in the constant-pass-energy mode at a resolution of $150 \mathrm{meV}$ separating photcelectrons corresponding to the different vibrational modes of the molecular ion. The Earth's magnetic field is reduced to less than $1 \%$ by means of three pairs of Helmholtz coils.

The photoelectron intensity in this geometrical arrangement is proportional to

$$
I(\theta) \propto 1+\frac{1}{4} \beta(1+3 P \cos 2 \theta)
$$


where $P$ is the degree of linear polarisation of the incident radiation and $\theta$ is the angle between the electric field vector and the momentum of the outgoing photoelectron. The asymmetry parameter $\beta$ characterises the deviation of the angular dependence of the electron intensity from isotropy and depends upon the dipole matrix elements and phaseshift differences of the transitions to continuum wavefunctions. Equation (1) holds for both partially linearly polarised and eliptically polarised radiation (Schmidt 1973). The asymmetry parameters of the angular intensity dependence are obtained from pairs of constant-ionic-state runs with the polarisation of the vuv radiation parallel $\left(\theta=0^{\circ}\right)$ and perpendicular $\left(\theta=90^{\circ}\right)$ to the momentum of the electrons accepted by the spectrometer. The asymmetry parameter $\beta$ can then be readily determined from the ratio $R$ of intensities $I\left(0^{\circ}\right) / I\left(90^{\circ}\right)$ and $P$, the degree of linear polarisation, according to:

$$
\beta=4(R-1) /[1-R+3 P(R+1)] .
$$

The results for the $\mathrm{HI}^{+2} \Pi_{3 / 2}\left(v^{\prime}=0\right)$ final state are presented in figure 2 . The intensities of the photoelectrons (figure $2(a), \theta=0^{\circ}$; figure $2(b), \theta=90^{\circ}$ ) are shown with error bars representing the single statistical error. The error bars of the asymmetry parameter $\beta$ (figure $2(c)$ ) contain the errors of the intensities and the uncertainty of the VUV polarisation.

The resonance structure observed results from the $\left(n_{\text {eff }}=7\right)$ members of Rydberg series HI $\left(5 \pi^{3}\right) n l \lambda$ converging to the ionisation limit of the ${ }^{2} \Pi_{1 / 2}\left(v^{\prime}=0\right)$ ionic state at $89119.5 \mathrm{~cm}^{-1}$ (Eland and Berkowitz 1977). The Rydberg states autoionise via spin-orbit interaction into the continuum leaving the ion in the ${ }^{2} \Pi_{3 / 2}\left(v^{\prime}=0\right)$ ground state. This autoionisation mechanism also occurs for the $5 \mathrm{p}$ ionisation of xenon, which is isoelectronic to HI. To distinguish between atomic and molecular effects a comparison can be made with the results for the $\mathrm{Xe}$ atom. Due to angular momentum selection rules, in atoms the excitation of a p electron leads to two Rydberg series: $n \mathrm{~s}, n \mathrm{~d}$. In xenon, the $n \mathrm{~d}$ series shows broad resonances on which the sharp $n$ s series is superimposed. Across the broad $n$ d resonance, a $\beta$ value of approximately 0.7 is measured, with $\beta$ approaching -0.7 at the minimum of the Fano profile (Samson and Gardner 1973).

The HI spectra are governed by the broad $\pi \rightarrow \mathrm{d} \delta, \mathrm{d} \pi$ transition with a similar structure for the asymmetry parameter $\beta$ as in xenon. Due to the reduced symmetry of the molecule, the angular momentum selection rules in molecular photoionisation lead to more transitions than in atoms. The strongest purely molecular feature in the spectrum occurs at $86570.0 \mathrm{~cm}^{-1}$. It may be assigned to a $\pi \rightarrow \mathrm{p} \pi$ or, possibly, a $\pi \rightarrow \mathrm{p} \sigma$ transition. This resonance gives a positive $\beta$ value superimposed on the maximum with negative $\beta$ values. The distance between two points in our measurements is approximately six times larger than the instrumental bandwidth. Thus the structure visible in our spectra is clearly not limited by a lack of resolution.

The autoionising Rydberg series were calculated by an ab initio MQDT technique (Lefebvre-Brion et al 1985). The calculation was performed not only for the cross section but also for the other dynamical parameters of the photoionisation process (asymmetry and spin-polarisation parameters) for the $\left(n_{\mathrm{eff}}=6\right)$ members of the Rydberg series. We have scaled these results to $\left(n_{\mathrm{eff}}=7\right)$ in order to compare the calculation with our experimental values. Furthermore, we evaluated the intensities for $\theta=90^{\circ}$ and $\theta=0^{\circ}$ from results of the calculation of the total cross section and the $\beta$ asymmetry parameter. The broken curves in figure 2 show the prediction of the calculation; it was shifted by $60 \mathrm{~cm}^{-1}$ to lower energies in order to achieve best agreement at the 

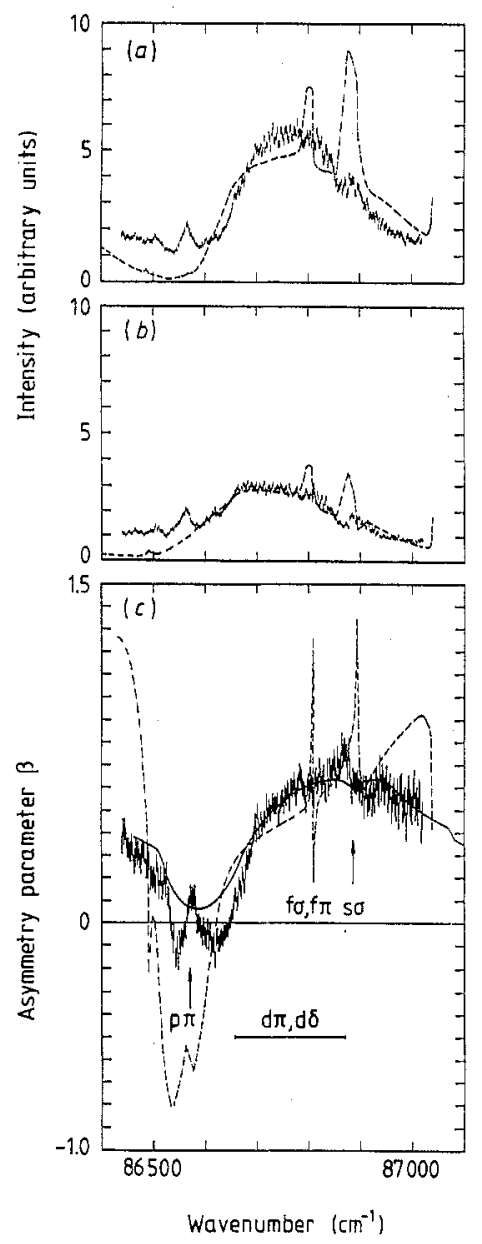

Figure 2. Results for the intensity and asymmetry parameter $\beta .(a)$ and $(b)$ show the electron intensities at $\theta=0^{\circ}$ and $\theta=90^{\circ}$, respectively. The resulting asymmetry parameter $\beta$ is given in $(c)$. The experimental data are denoted by their error bars. The broken curves are the results of an ab initio calculation by Lefebvre-Brion et al (1985); the classification of the resonances is also taken from this reference. The full curve in $(c)$ shows the result of a measurement with synchrotron radiation (Carlson et al 1986).

resonance positions. In the calculation an $a b$ initio ionisation potential was used, which differs from the experimentally known value. The amount of the shift is the same for both intensities and the $\beta$ parameter. The classification of the resonances is taken from the calculation.

The calculation shows a reasonable agreement with our measurements, although some details, especially the predicted sharp resonances, are rather weak (and smeared out) in the experiment. Except for a test calculation for $l$ uncoupling at $J=4$, no rotational effects are taken into account in the calculation. Since our molecular beam is close to room temperature, at least ten rotational levels of the ground state are significantly populated with the maximum at $J=4$. This should lead to a smearing out of any sharp structure, since the intensity is distributed over the different possible rotational transitions. Furthermore, $l$ - and $s$-uncoupling effects occuring for higher $J$ 
states lead to transitions differing from the case assumed in the calculation (a molecule without rotation). These effects can be seen in photoelectron yield spectra (Hart and Hepburn 1989).

For comparison, in figure $2(\mathrm{c})$ the results of measurements with synchrotron radiation (Carlson et al 1986), are shown as full curve $\dagger$. In direct comparison with our results, one can see the influence of the radiation bandwidth on the fine structure of the spectrum. For example, at $86570.0 \mathrm{~cm}^{-1}$ only the present data show a pronounced resonance feature. Nevertheless, the general shape obtained in photoionisation with coherent and synchrotron radiation is in excellent agreement.

We presented first results of a new experimental technique to study the dynamics of molecular photoionisation. The results show better resolved structure than previous methods could achieve. Theoretical calculations of dynamical parameters in the photoionisation process have to be improved by taking into account the influence of molecular rotation in order to compare with the experimental results more closely. One experimental approach to search for better agreement with calculation would be the use of a supersonic molecular beam to cool the rotation of the molecules.

We thank $\mathrm{R}$ Wallenstein, $\mathrm{H}$ Lefebvre-Brion and $\mathrm{G}$ Raseev for valuable discussions. The assistance of $\mathrm{H} \mathrm{W}$ Klausing in the set-up of the spectrometer is gratefully acknowledged. This work was supported by the Deutsche Forschungsgemeinschaft (SFB 216) and the European Commission.

\section{References}

Carlson T A, Gerard P, Krause M O, von Wald G, Taylor J W and Grimm F A 1986 J. Chem. Phys. 84 4755-9 Eland J H D and Berkowitz J 1977 J. Chem. Phys. 67 5034-9

Hart D J and Hepburn J W 1989 Chem. Phys. 129 51-64

Hilbig R and Wallenstein R 1983 IEEE J. Quantum Electron. 19 1759-70

Huth T, Mank A, Böwering N, Schönhense G, Wallenstein R and Heinzmann U 1988 Electronic and Atomic Collisions (London: Elsevier) pp 607-12

Jost K 1979 J. Phys. E: Sci. Instrum. 12 1006-12

Lefebvre-Brion H, Giusti-Suzor A and Raseev G 1985 J. Chem. Phys. 83 1557-66

Lucas C B 1973 J. Phys. E: Sci. Instrum. 6 991-4

Samson J A R and Gardner J L 1973 Phys. Rev. Lett. 31 1327-30

Schmidt V 1973 Phys. Lett. 45A 63-4

$\uparrow$ The data were taken from figure 1 of Carlson et al. Since figure 1 and figure 3 of this reference differ in energy scale, we have shifted the scale by $150 \mathrm{~cm}^{-1}$ to higher energies for comparison with our data. 